\title{
Sensibilidad y especificidad de tres métodos complementarios para el diagnóstico de síndrome de túnel carpiano
}

\section{Sensitivity and specificity of three complementary methods in the diagnosis of carpal tunnel syndrome}

\author{
Cristiani-Winer M,** Allende-Nores C,* Aguirre GL,* Robles CO,* Ré R*
}

Instituto Allende de Cirugía Reconstructiva. Córdoba, Argentina.

RESUMEN. Introducción: Actualmente, no existe un estándar de referencia aceptado universalmente para el diagnóstico del síndrome de túnel carpiano, por lo que se le considera una patología de «clase latente», es decir, que carece de alguna prueba diagnóstica que dé certeza absoluta de la presencia de la enfermedad. Métodos: Estudio prospectivo, observacional y analítico en el cual se evaluaron los tres métodos diagnósticos utilizados para el síndrome de túnel carpiano (examen clínico, ecografía y electromiografía). En él, se establecieron valores de normalidad para cada método diagnóstico. Resultados: Se evaluó un total de 50 personas (14 hombres y 36 mujeres). El examen clínico presentó correlación positiva y significativa con el diámetro del nervio mediano afectado (ecografía) $(\mathrm{R}=0.694$ y $\mathrm{p}=0.032)$. Los valores del área bajo la curva (AUC, por sus siglas en inglés) para la velocidad de conducción del nervio mediano (VCNM), velocidad de conducción de nervio cubital (VCNC) y latencia distal motora (LDM) por electromiografía fueron de 0.60, $0.519 \mathrm{y}<0.50$, respectivamente, lo cual determinó que el valor diagnóstico de las características por electromiografía es malo. Conclusión: Nuestro trabajo establece al examen clínico como una herramienta de buena calidad, siendo el método más sensible para el diagnóstico de síndrome de túnel carpiano. Si existiera la necesidad de realizar una confirmación diagnóstica, la ecografía mostró ser un estudio altamente satisfactorio, el cual genera menos estrés, dolor e invasión al paciente, disminuyendo, a su vez, el gasto de salud y agilizando el proceso.

Palabras clave: Túnel carpiano, métodos complementarios, diagnóstico, precisión.
ABSTRACT. Introduction: Currently there is no universally accepted standard of reference for the diagnosis of carpal tunnel syndrome so it is considered a «latent class» pathology, that is, it lacks any diagnostic tests that absolutely certain the presence of disease. Methods: Prospective, observational and analytical study evaluating the three diagnostic methods used for carpal tunnel syndrome (clinical examination, ultrasound and electromyography). Normality values were set for each diagnostic method. Results: 50 people (14 men and 36 women) were evaluated. The clinical examination showed a positive and significant correlation with the diameter of the affected median nerve (ecography) $(\mathrm{R}=0.694, \mathrm{p}=0.032)$. The values of the under the curve area (UCA) for median nerve conduction speed (MNCS), cubital nerve conduction speed (CNCS), and distal latency (DL) by electromyography were 0.60 , 0.519 and less than 0.50 respectively. This states that the diagnostic value of electromyography characteristics is bad. Conclusion: Our work establishes clinical examination as a good quality tool being the most sensitive method for diagnosing carpal tunnel syndrome. If there was a need for diagnostic confirmation, the ultrasound proved to be a highly satisfactory study generating less stress, pain and invasion to the patient, decreasing health expenditure and speeding up the process.

Keywords: Carpal tunnel, complementary methods, diagnosis, accuracy.

Nivel de evidencia: Estudio prospectivo. III

* Cirujano ortopedista.

Dirección para correspondencia:

Mauro Cristiani-Winer

Independencia 757, 1er. piso, Córdoba 5000, Argentina.

Tel: +543515736330

E-mail: maurocw7@gmail.com 


\section{Introducción}

El síndrome de túnel carpiano (STC) es, a nivel de la extremidad superior, una de las patologías del aparato musculoesquelético más frecuente. Éste se define como una neuropatía por compresión del nervio mediano a nivel de la muñeca, el cual provoca una alteración en la función del nervio. . $^{1,2,4,5}$

A lo largo de los años, se han realizado intensos avances con respecto al diagnóstico y tratamiento del STC. Por ejemplo, Graham y colaboradores desarrollaron una herramienta para el diagnóstico clínico de la patología, haciendo hincapié en seis hallazgos que provienen de la historia clínica del paciente y del examen físico. Estos criterios clínicos se definieron debido a la preponderancia que tenían en los exámenes físicos y la influencia importante sobre la probabilidad de diagnosticar STC bajo estos hallazgos.,.$^{2,3}$

Durante muchos años, por un lado, los estudios de conducción nerviosa cobraron gran relevancia en el diagnóstico de STC, sin embargo, luego se demostró que existe entre 16 y $34 \%$ de falsos negativos, por lo que siempre el diag- nóstico toma los hallazgos clínicos como patrón de referencia. ${ }^{1,2,3,4}$ Por otro lado, la ecografía se presenta como una opción diagnóstica con similar sensibilidad y especificidad que los estudios de conducción nerviosa, pero con menores exigencias para el paciente y más rentables para el sistema de salud.

Actualmente, no existe un estándar de referencia aceptado universalmente para el diagnóstico de STC, por lo que se le considera que es una patología de "clase latente», es decir, que se carece de alguna prueba diagnóstica que dé certeza absoluta de la presencia de enfermedad. A partir de este razonamiento, el objetivo de nuestro trabajo es realizar una comparación entre el examen físico, la ecografía y la electromiografía.

\section{Material y métodos}

Se realizó un estudio prospectivo, observacional y analítico en el que se evaluaron tres métodos diagnósticos utilizados para el síndrome de túnel carpiano. Éstos son el cuestionario de opiniones CTS-6 (Figura 1), la ecografía
Apellido y nombre:

DNI:
Edad:

Sexo: $\mathrm{M} / \mathrm{F}$

\section{Evaluación clínica CTS-6}

Tomado y modificado de: Graham B. The value added by electrodiagnostic testing in the diagnosis of carpal tunnel syndrome. J Bone Joint Surg Am. 2008; 90: 2587-93.

Historia y síntomas

1. Parestesia predominante o exclusivamente en el territorio del nervio mediano

(Los síntomas sensoriales se localizan principalmente en los dedos pulgar, índice y medio)

2. Parestesia nocturna

(Los síntomas interrumpen el sueño del paciente)

Examen físico

3. Atrofia y/o debilidad en región tenar

(El volumen del área tenar se reduce y/o las pruebas motoras manuales muestran pérdida de la resistencia)

4. Prueba de Phalen positiva

5 ptos.

(La flexión sostenida de la muñeca produce empeoramiento de los síntomas)

5. Pérdida de discriminación de 2 puntos

5 ptos.

(Falla al discriminar 2 puntos mantenidos a $5 \mathrm{~mm}$ a menos el uno del otro, inervados por el mediano)

6. Signo de Tinel

4.5 ptos.

(Ligero tapping sobre nervio mediano a nivel del túnel carpiano causando parestesias radiales)

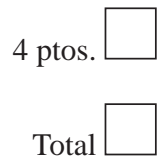

Figura 1: Cuestionario de opiniones CTS-6 para evaluación clínica de síndrome de túnel carpiano. 
Tabla 1: Estadística descriptiva de las características evaluadas en la muestra de pacientes.

\begin{tabular}{|c|c|c|c|c|c|}
\hline Variable & Media & Error estándar & P25 & P75 & P90 \\
\hline \multicolumn{6}{|l|}{ Evaluación clínica (puntaje) } \\
\hline Varones & 15.75 & 5.82 & 11.5 & 21 & 22.5 \\
\hline Mujeres & 16.34 & 3.72 & 13 & 17.5 & 21 \\
\hline \multicolumn{6}{|l|}{ Diámetro nervio mediano afectado (mm) } \\
\hline Varones & 14.38 & 2.58 & 13 & 16 & 17 \\
\hline Mujeres & 14.01 & 2.37 & 12.25 & 15 & 17 \\
\hline \multicolumn{6}{|l|}{ Diámetro nervio mediano contralateral (mm) } \\
\hline Varones & 7.58 & 0.86 & 7 & 8 & 9 \\
\hline Mujeres & 8.26 & 1.06 & 9 & 9 & 9.5 \\
\hline Velocidad de conducción de nervio mediano* & 41.33 & 6.56 & 37.4 & 44.8 & 49.57 \\
\hline Velocidad de conducción de nervio cubital* & 57.54 & 5.67 & 53.4 & 60.9 & 64.75 \\
\hline Latencia distal motora* & 5.17 & 1.29 & 4.2 & 5.72 & 6.165 \\
\hline Velocidad de conducción de nervio mediano** & 53.97 & 7.32 & 51.7 & 57.63 & 64.07 \\
\hline Velocidad de conducción de nervio cubital** & 56.68 & 5.85 & 51.04 & 62.9 & 69.75 \\
\hline Latencia distal motora** & 4.09 & 1.59 & 3.82 & 5.86 & 6.49 \\
\hline
\end{tabular}

bilateral de muñeca y la electromiografía de miembros superiores. Para este estudio, se trabajó conjuntamente con el Servicio de Diagnóstico por Imágenes y Neurología.

Los criterios de inclusión fueron: pacientes $>18$ años, de ambos sexos y con conducta quirúrgica de STC. Los criterios de exclusión fueron neuropatía periférica conocida y cirugía previa de STC.

En primera instancia, se definió que la posibilidad de diagnóstico clínico de STC sería $>25 \%$ cuando el puntaje de la valoración sea $\geq 5$ respecto al CTS- 6 y $>80 \%$ con un puntaje $>12 .^{3}$ Desde el punto de vista ecográfico, se consideró STC en todo aquel paciente que presentó un diámetro del nervio mediano $\geq 10 \mathrm{~mm}$ y que, al compararlo con una muñeca contralateral, debiera ser $<10 \mathrm{~mm}$ para considerarlo sano.

Respecto a la electromiografía, se consideraron tres valores en cada miembro superior para comparar entre sí: velocidad de conducción de nervio mediano desde muñeca a dedo índice (función sensitiva), velocidad de conducción de

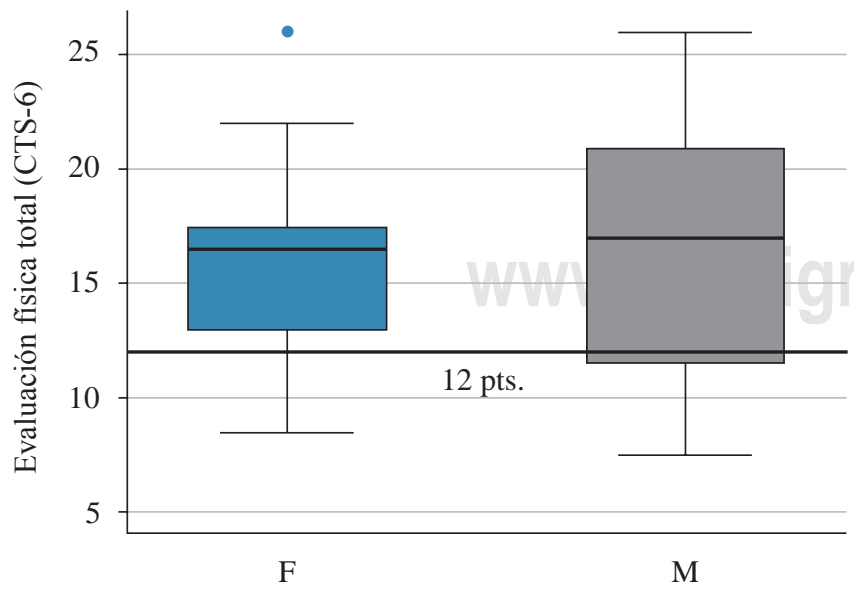

nervio cubital desde muñeca a dedo meñique (función sensitiva del nervio de referencia) y latencia distal de nervio mediano en muñeca a abductor corto del pulgar (función motora). Los parámetros de normalidad para la valoración sensitiva no debían presentar un valor $<50 \mathrm{~m} / \mathrm{s}$ ni una diferencia $>10 \mathrm{~m} / \mathrm{s}$ entre el nervio mediano y el nervio cubital; la valoración motora tampoco debía exceder una latencia distal de 4 milisegundos.

Previo a comenzar con los diferentes estudios diagnósticos, se entregó a cada paciente el consentimiento informado avalado por el Comité de ética de la institución en el cual se explicaba el propósito del trabajo; con ello, se buscó que el paciente pudiera decidir si aceptaba o no formar parte del estudio.

\section{Resultados}

La muestra de pacientes estuvo compuesta por 50 personas (14 varones y 36 mujeres), cuya edad promedio fue

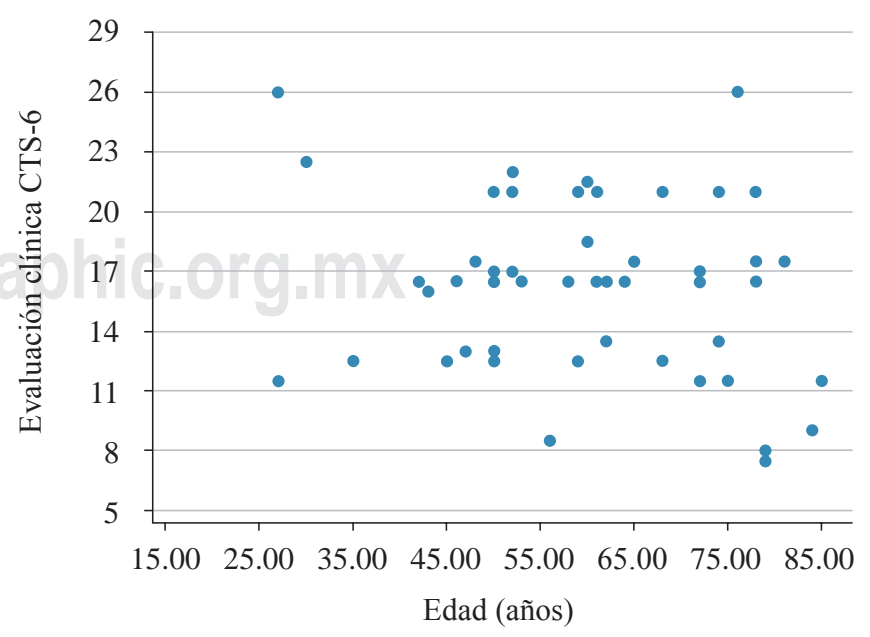

Figura 2: Diagrama de caja de la variable evaluación clínica del CTS-6 según sexos (izquierda) y diagrama de dispersión entre esta característica y la edad. 


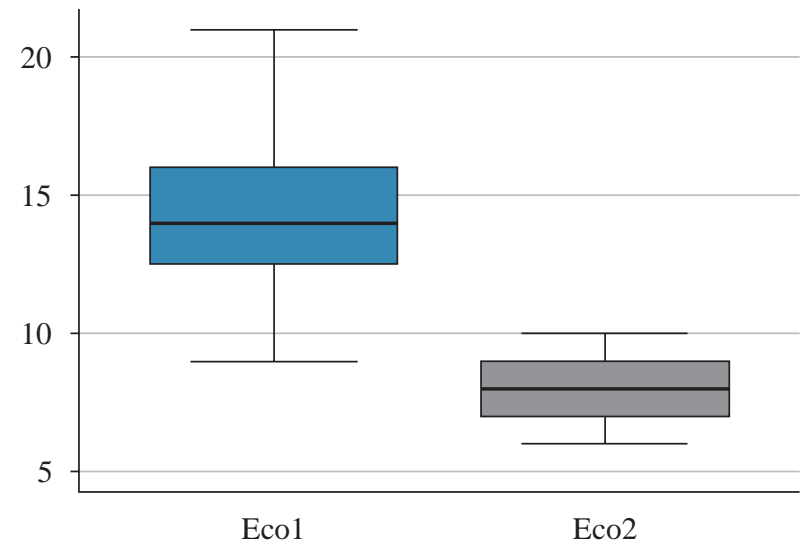

Figura 3: Diagramas de caja del diámetro del nervio mediano afectado (Eco1) y no afectado (Eco2).

de 54.92 años para hombres y de 69.8 años para mujeres, con distribuciones estadísticamente diferentes $(\mathrm{p}=0.0012)$. Sólo $10 \%$ de los hombres tuvieron edades inferiores a los 60 años, en tanto que en las mujeres, prácticamente, $75 \%$ estuvo por debajo de esa referencia. La Tabla 1 presenta el resumen de las distribuciones empíricas de las principales características estudiadas.

El índice de evaluación clínica (EC), que se compone de seis ítems, tuvo un valor central (EE) igual a 16.2 (0.62); su distribución, cuyo percentil 25 (P25) fue de 11.9, no mostró diferencias entre sexos $(\mathrm{p}=0.668)$, aunque sí una marcada asociación positiva con la edad ( $\mathrm{p}=0.0247)$. La Figura 2 ilustra estos aspectos, observándose que en el diagrama de

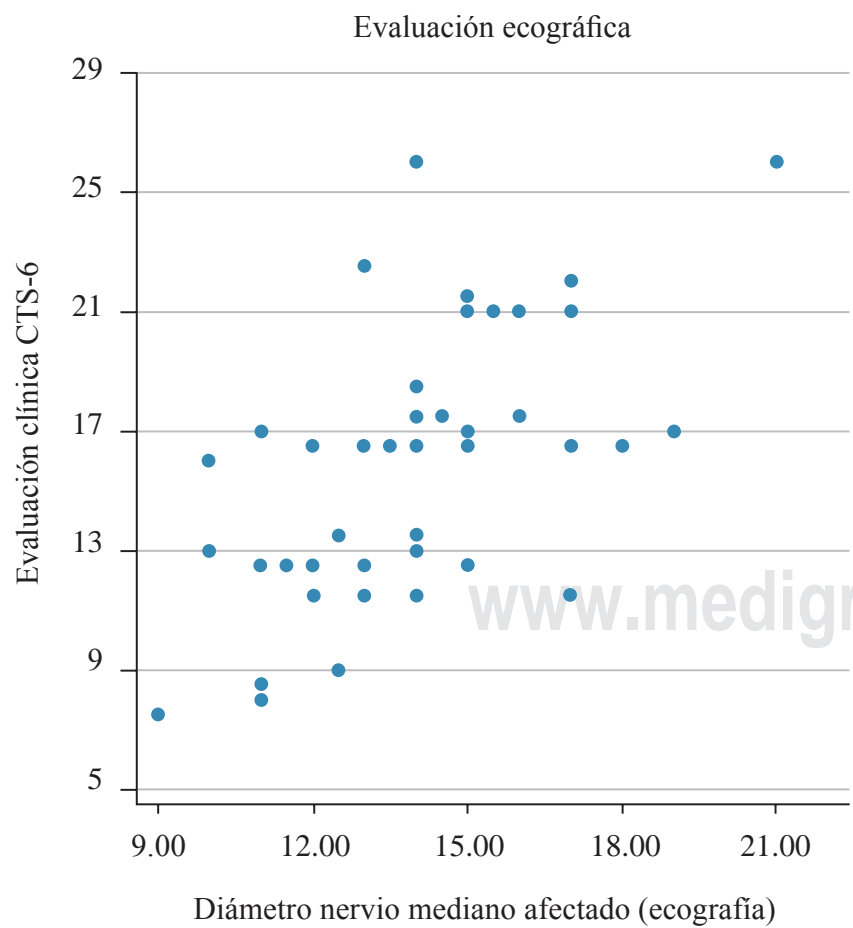

cajas (izquierda) se introdujo una línea de corte en el valor de EC igual a 12, el cual es considerado el punto límite para el diagnóstico recomendado de síndrome de túnel carpiano, ya que, como se dijo anteriormente, éste representa $80 \%$ de posibilidades de padecer la enfermedad; $84 \%$ de pacientes superó ese valor límite, lo cual estuvo asociado con el sexo $(\mathrm{p}=0.047: 78 \%$ para mujeres y $22 \%$ para varones).

La Tabla 1 también presenta los valores medios del diámetro del nervio mediano afectado y del correspondiente contralateral, cuya distribución es ilustrada en la Figura 3 y cuyas diferencias fueron altamente significativas $(\mathrm{p}=$ 0.001), observándose valores superiores de esta característica en la mano afectada. En términos generales, el diámetro observado del nervio mediano afectado superó el valor de referencia ( $10 \mathrm{~mm}$; $\mathrm{p}<0.001)$, incluso, en sus percentiles menores y el semejante pero contralateral. Por el contrario, este diámetro presentó una distribución empírica con valores inferiores a $10 \mathrm{~mm}(\mathrm{p}<0.02)$. El sexo no estuvo asociado con esta diferenciación ( $\mathrm{p}=0.624)$.

La evaluación clínica presentó una correlación positiva y significativa con el diámetro del nervio mediano (afectado) $(\mathrm{R}=0.694 ; \mathrm{p}=0.032)$. La Figura 4 muestra la asociación directa entre estas dos valoraciones en la muestra de pacientes.

En aquellos pacientes cuya EC superó el puntaje de 12, el valor medio del diámetro del nervio mediano afectado fue 14.45 (DE 2.31), mientras que aquéllos correspondientes al referente contralateral fue de 8.15 (DE 1.03). Para el grupo de pacientes con un puntaje de EC inferior a 12, fue significativamente diferente en el nervio afectado con $12.61(\mathrm{DE}$

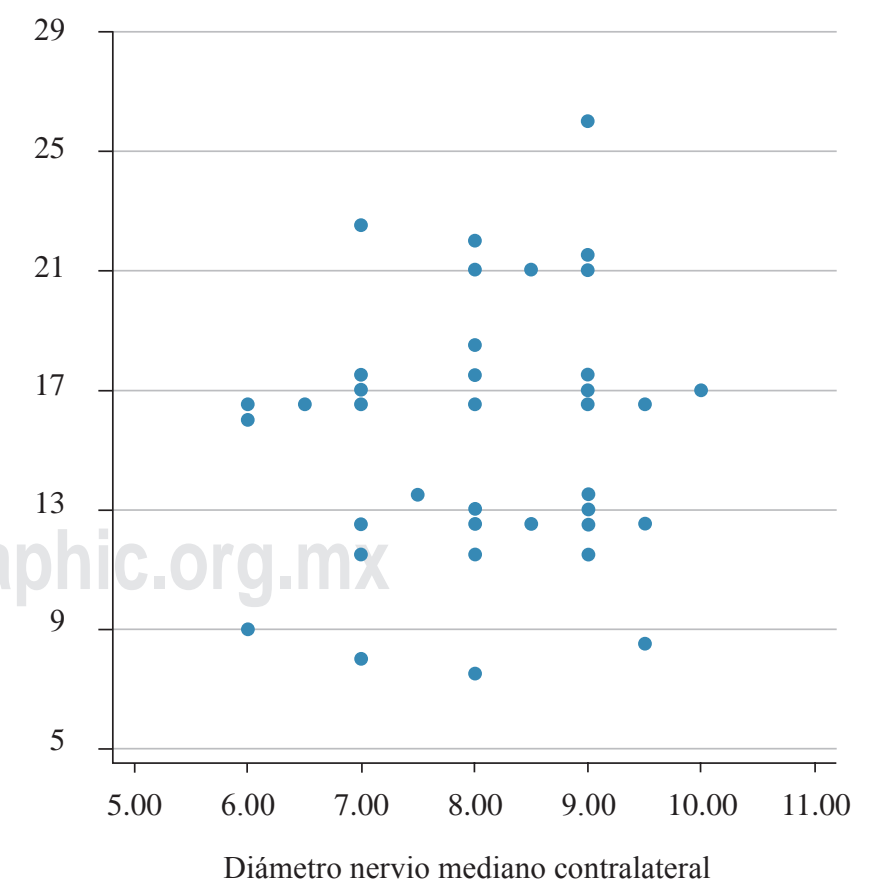

Figura 4: Diagramas de dispersión entre el diámetro del nervio mediano afectado (izquierda) y no afectado (derecha) y la evaluación clínica en función de los seis scores. 
$2.29 ; \mathrm{p}=0.0354)$ y en el no afectado con $7.63(\mathrm{DE} 1.09 ; \mathrm{p}$ $=0.206$ ).

El puntaje que valora clínicamente a los pacientes (EC) tuvo un desempeño satisfactorio en cuanto a lo obtenido por el examen por ecografía del nervio mediano. La Figura 5 ilustra las tendencias de especificidad y sensibilidad del EC respecto a las imágenes de partes blandas (izquierda) y la curva de rendimiento diagnóstico (aplicación sanitaria de la curva ROC, [Receiver Operating Characteristic]). En la Figura 5, también se muestra que la relación sensibilidad/ especificidad entre el EC y la ecografía es ampliamente satisfactoria, ya que el punto de corte alcanza un valor de probabilidad cercano a 0.80 .

El área bajo la curva (AUC, por sus siglas en inglés) estimada fue 0.836 , lo que indica un valor diagnóstico bueno $(0.5<$ AUC $<1$, donde 1 representa un valor diagnóstico perfecto y 0.5 es una prueba sin capacidad discriminatoria diagnóstica). La literatura establece un consenso al respecto, pues determina que para $0.5,0.6$ : test malo; $0.6,0.75$ : test regular y $0.75,0.9$ : test bueno. Así, la prueba diagnóstica utilizando EC indicó que existe aproximadamente $84 \%$ de probabilidad de que el diagnóstico realizado a un paciente sea más correcto que el de una persona sana escogida al azar.

Las características obtenidas mediante electromiografía para evaluar la conducción nerviosa, como la velocidad de conducción de nervio mediano (VCNM), del nervio cubital (VCNC) y de la latencia distal motora (LDM) de la muñeca con sospecha de STC son presentadas en la Tabla 1. Se observa que la correlación esperada entre las dos primeras se atenúa $(\mathrm{p}=0.034)$ cuando se evalúa en la muñeca con sospecha de STC: $\mathrm{R}=0.245$ en comparación con $\mathrm{R}=0.49$ que corresponde a la muñeca sin sospecha.

Los valores de referencia considerados normales para las variables VCNM y VCNC por electromiografía fue > $50 \mathrm{~m} / \mathrm{s}$. La VCNM promedio fue significativamente menor

Diámetro nervio mediano afectado vs evaluación clínica CTS-6

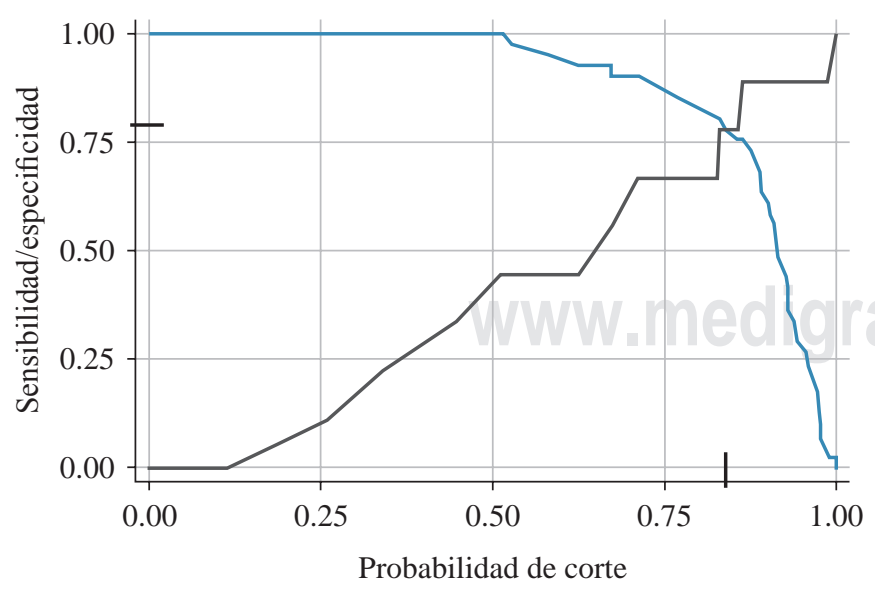

Sensibilidad - Especificidad a $50(\mathrm{p}<0.001)$ en la muestra de todos los pacientes, pero no mostró diferencias significativas entre los diagnosticados con STC por la EC $(\mathrm{p}=0.495)$. Por otro lado, la VCNC fue, en promedio, superior al valor normal $(50 \mathrm{~m} / \mathrm{s})$ con un intervalo de confianza $95 \%\left(\mathrm{IC}_{95 \%}\right)$ igual a $55.92 ; 59.16 \mathrm{~m} / \mathrm{s}$, mostrando también diferencias nulas entre los diagnosticados con STC por la valoración clínica $(\mathrm{p}=0.904)$. El mismo comportamiento fue para la latencia distal motora: los pacientes reportaron valores dentro del rango no normal con un $\mathrm{IC}_{95 \%}$ 4.81; 5.54 y sin diferencias entre los dos grupos $(p=0.44)$. Si bien, no se encontraron diferencias significativas, es interesante mostrar que los pacientes con VCNM mayor a 50 (normal) tuvieron un $\mathrm{IC}_{95 \%}$ igual a $3.54 ; 5.63$, esto es, prácticamente $95 \%$ de los sujetos reportaron valores de LDM superiores a lo establecido como normal. La Figura 6 ilustra las distribuciones de las variables descriptas.

Los valores del AUC para VCNM, VCNC y LDM por electromiografía fueron $0.60,0.519$ y menor a 0.50 , respectivamente, lo cual establece que el valor diagnóstico de las características conjuntas por electromiografía es malo.

\section{Discusión}

El diagnóstico del STC es un tema controversial, el cual continúa en investigación. A pesar de los diferentes avances, no existe un estándar de oro que confirme el diagnóstico con una perfecta sensibilidad y especificidad. El STC además representa una de las patologías más costosas, debido a que tiene gran prevalencia en la población trabajadora, por lo que no sólo se deben afrontar los gastos propios del tratamiento médico, sino también a los costos por compensación de los trabajadores, pérdida de la productividad y pérdida de salarios, entre otros. ${ }^{1,2,3}$

Durante mucho tiempo, los estudios de conducción nerviosa se han considerado como el estándar de referencia

Figura 5: Sensibilidad y especificidad de EC comparado con el examen por ecografía del nervio mediano afectado (izquierda) y curva ROC para el rendimiento diagnóstico (derecha). 

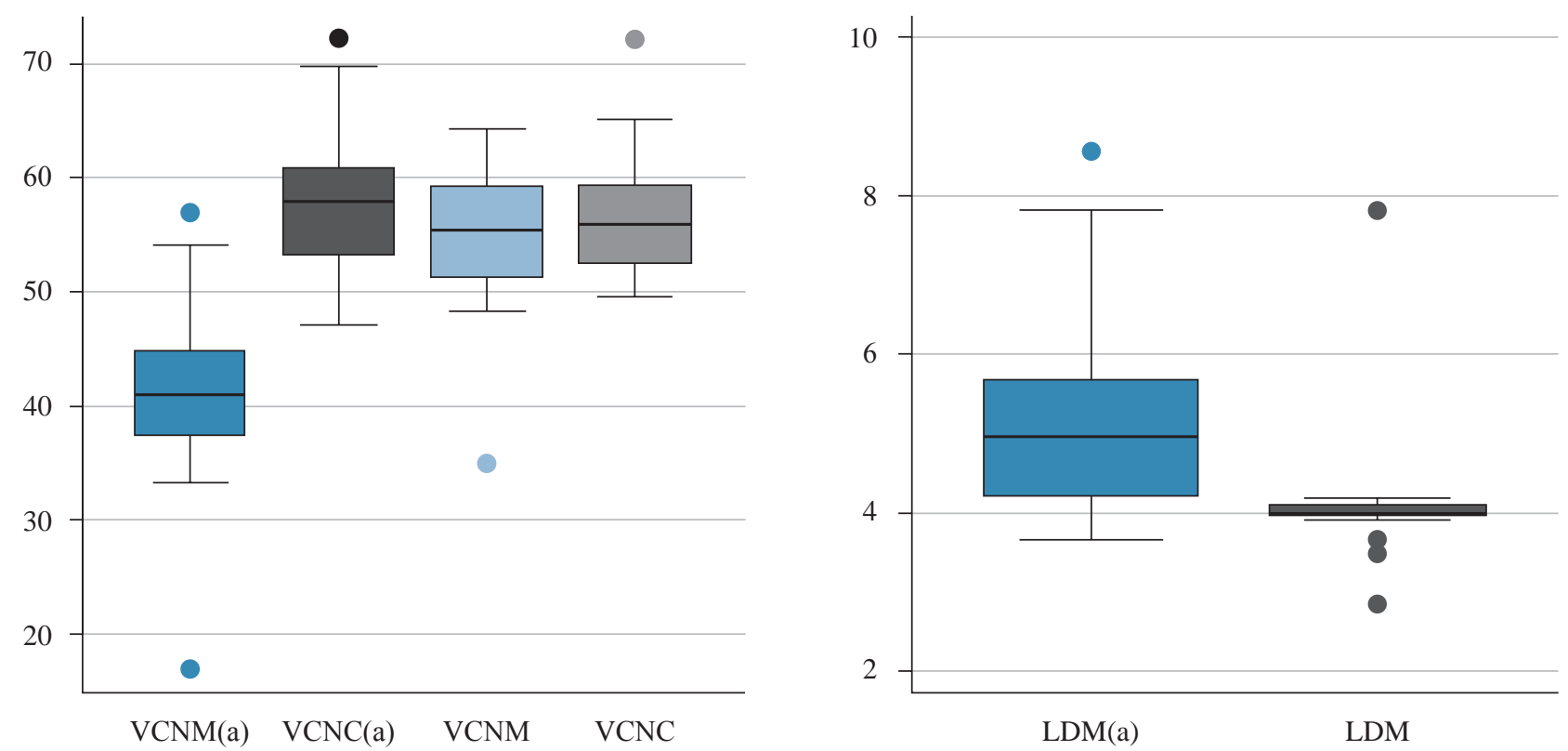

Figura 6: Diagramas de caja de las variables obtenidas por electromiografía, en la muestra de pacientes y para ambas muñecas (afectada y no afectada).

para confirmar el STC. Sin embargo, la verdadera precisión diagnóstica de los estudios de conducción nerviosa ha sido difícil de determinar. ${ }^{1,2,3,4,5,6,7,8}$ Mediante nuestro trabajo, demostramos que el examen clínico presentó una correlación positiva y significativa con el diámetro del nervio mediano afectado (ecografía) $(\mathrm{R}=0.694 ; \mathrm{p}=0.032)$, permitiendo ser suficiente para realizar el diagnóstico de STC. Sin embargo, debido a los valores del AUC para VCNM, VCNC y LDM por electromiografía, el valor diagnóstico de este estudio fue malo $(0.60,0.519 \mathrm{y}<0.50$, respectivamente).

En los últimos años, diferentes autores como Graham y colaboradores o Fowler y su equipo han estudiado la precisión clínica y ecográfica en el STC. ${ }^{1,2,3}$ Estos últimos autores, en un trabajo realizado en 2015, encontraron precisión diagnóstica similar entre la ecografía y la conducción nerviosa, siempre y cuando se haya utilizado una herramienta clínica validada como estándar de referencia. La especificidad fue mayor en la ecografía que en el estudio de conducción nerviosa con un valor predictivo muy alto (99\%), lo cual se asemeja con nuestros resultados $(97 \%))^{1,6}$

El uso de la ecografía tiene varias ventajas respecto a los estudios de conducción nerviosa. En primer lugar, es un estudio no invasivo, por lo que se evita la incomodidad, el dolor y el estrés que se requieren al someterse a estímulos eléctricos repetidos y la colocación de agujas repetidamente durante el estudio. En segundo lugar, la diferencia en los tiempos para realizar cada uno de los estudios es importante, ya que la ecografía es rápida ( 2 a 5 minutos) respecto de las pruebas electrodiagnósticas (entre 30 y 45 minutos). Tercero, existe una importante diferencia en el gasto de salud entre ambos estudios, siendo la ecografía la más económica. Por último, la ecografía tiene la capacidad de aportar información extra respecto a la causa del STC, ya que pue- de poner en evidencia diferentes compresiones que pueden producir la sintomatología (presencia de quistes o tumoraciones, entre otras). ${ }^{6,7,9}$

Carril y colaboradores realizaron una encuesta sobre el diagnóstico y tratamiento del STC en miembros de la Sociedad Americana de Cirugía de Mano, encontrando que 72\% de respuestas aconsejan la liberación del túnel carpiano a partir del examen físico y el cese completo de la sintomatología luego de realizar infiltración con corticoides de depósito; $47 \%$ respondió que las pruebas electrodiagnósticas rara vez (o nunca) son necesarias para indicar la cirugía. Por último, 57\% respondió que, en caso de solicitar algún estudio, esto se debía a posibles cuestiones medicolegales. ${ }^{10,11}$

D'Auria y su equipo de investigación compararon el examen clínico con la electromiografía. En este estudio, mostraron que los cirujanos subespecialistas en cirugía de mano no requieren de estudios de conducción nerviosa para diagnosticar el STC y que el examen clínico brindó una precisión cercana a 90\%. También demostraron que la experiencia del cirujano y su longevidad en la práctica clínica mejoran la precisión diagnóstica. ${ }^{12}$

En un estudio realizado por Sears y otros, se determinó que los pacientes sometidos a electromiografía esperaron $36 \%$ más de tiempo entre el diagnóstico y el acto quirúrgico con una consulta adicional y un gasto promedio de 996 dólares mayor. Las infiltraciones con corticoides también se relacionaron con una demora entre el diagnóstico y la cirugía, pero con un costo adicional de sólo un tercio del producido por la electromiografía. . $^{13,14}$

Como conclusión, nuestro trabajo aporta evidencia novedosa para la clínica porque la establece como una herramienta de diagnóstico de buena calidad, pudiendo ser suficiente para el paciente. Sin embargo, si existiera la nece- 
sidad de realizar una confirmación diagnóstica, la ecografía mostró ser un estudio altamente satisfactorio, el cual genera menos estrés, dolor e invasión al paciente, disminuye el gasto de salud y agiliza el proceso, ya que se realiza en unos pocos minutos.

Como limitaciones de nuestro trabajo, se puede destacar la falta de un grupo control sano, debido a que no sería apropiado someter a gente sin sospecha de STC a realizarse estudios invasivos de electrodiagnóstico. De igual manera, sucedió en el examen clínico, donde se realizó el CTS-6 a los pacientes que consultaban con sintomatología, lo que nos permitía sospechar la presencia de STC.

Bibliografía

1. Fowler JR, Cipolli W, Hanson T. A comparison of three diagnostic tests for carpal tunnel syndrome using latent class analysis. $J$ Bone Joint Surg Am. 2015; 97(23): 1958-61.

2. Graham $B$. The value added by electrodiagnostic testing in the diagnosis of carpal tunnel syndrome. J Bone Joint Surg Am. 2008; 90(12): 2587-93.

3. Graham B, Regehr G, Naglie G, Wright JG. Development and validation of diagnostic criteria for carpal tunnel syndrome. $J$ Hand Surg Am. 2006; 31(6): 919-24.

4. Calandruccio JH, Thompson NB. Carpal tunnel syndrome: making evidence-based treatment decisions. Orthop Clin North Am. 2018; 49(2): 223-9.

5. Duckworth AD, Jenkins PJ, McEachan JE. Diagnosing carpal tunnel syndrome. J Hand Surg Am. 2014; 39(7): 1403-7.

6. Fowler JR, Maltenfort MG, Ilyas AM. Ultrasound as a first-line test in the diagnosis of carpal tunnel syndrome: a cost-effectiveness analysis. Clin Orthop Relat Res. 2013; 471(3): 932-7.
7. Day CS. An alternative solution for the diagnosis of carpal tunnel syndrome: commentary on an article by John R. Fowler, MD, et al. "A comparison of three diagnostic tests for carpal tunnel syndrome using latent class analysis”. J Bone Joint Surg Am. 2015; 97(23): e78.

8. American Academy of Orthopaedic Surgeons [portal web]. Clinical guideline on diagnosis of carpal tunnel syndrome. Rosemont (IL): American Academy of Orthopaedic Surgeons (AAOS); 2007. [Accesado el 28 de julio de 2014] Disponible in: http://www.aaos.org/ Research/guidelines/CTS_guideline.pdf.

9. Kwon BC, Jung KI, Baek GH. Comparison of sonography and electrodiagnostic testing in the diagnosis of carpal tunnel syndrome. $J$ Hand Surg Am. 2008; 33(1): 65e71.

10. Lane LB, Starecki M, Olson A, Khon N. Carpal tunnel syndrome diagnosis and treatment: a survey of members of the American Society for Surgery of the Hand. J Hand Surg Am. 2014; 39: 2181-7.

11. Atroshi I, Flondell M, Hofer M, Ranstam J. Methylprednisolone injections for the carpal tunnel syndrome: a randomized, placebocontrolled trial. Ann Intern Med. 2013; 159: 309-17.

12. D'Auria JL, Montanez A, Toirac A, Goitz RJ, Fowler JR. Accuracy of surgeon diagnosis in predicting carpal tunnel syndrome Hand (NY). 2019; 1558944719856105 [published online ahead of print, 2019 Jun $18]$.

13. Sears ED, Swiatek PR, Hou H, Chung KC. Utilization of preoperative electrodiagnostic studies for carpal tunnel syndrome: an analysis of national practice patterns. $J$ Hand Surg Am. 2016; 41(6): 665-672.e1.

14. Sears ED, Lu YT, Wood SM, Nasser JN, Hayward R, Chung KC, et al. Diagnostic testing requested before surgical evaluation for carpal tunnel syndrome. J Hand Surg Am. 2017; 42(8): 623-629.e1.

Fuente de financiamiento: no hubo ninguna fuente de financiamiento para la realización de este trabajo. Los estudios complementarios que se evaluaron son estudios de rutina que se les realiza a pacientes que consultan con la sintomatología sugestiva de STC. 